\title{
Jardines que la Comunidad de Madrid ha perdido
}

\author{
Carmen Ariza Muñoz*
}

\section{RESUMEN:}

Aunque se hayan perdido algunos importantes jardines, en la actualidad la Comunidad de Madrid cuenta con un mayor números de ellos. Sírvannos como ejemplos de los que ya no se conservan el primer jardín Botánico de

la capital, pero en cambio existen el del paseo del Prado o los recientes de las Ciudades Universitarias de Madrid $y$ de Alcalá de Henares. Lo mismo

podría decir de los Jardines de Recreo, puesto que no nos han llegado los hechos en el siglo XIX, pero cada día surgen nuevos Parques de Atracciones y Temáticos (como el que se está realizando en San Martín

de la Vega). Imnumerables son también los de las viviendas privadas o los parques públicos, que aumentan progresivamente. Sin embargo, si han desaparecido o han cambiado de función casi todos los Reales Sitios, que surgieron, entre los siglos XVI Y $X I X$, como consecuencia de que Madrid fuera elegida, en 1561, capital del reino por Felipe II. Ésta es la causa de que nuestra Comunidad, siendo la cuarta más pequeña de España, cuente con casi la mitad de los jardines declarados históricoartísticos en nuestro país.

\section{SUMMARY:}

Aunque se hayan perdido algunos importantes jardines, en la actualidad la Comunidad de Madrid cuenta con un mayor números de ellos. Sívannos como ejemplos de los que ya no se conservan el primer jardín Botánico de la capital, pero en cambio existen el del paseo del Prado o los recientes de las Ciudades Universitarias de Madrid y de Alcalá de Henares. Lo mismo podría decir de los Jardines de Recreo, puesto que no nos han llegado los hechos en el siglo XIX, pero cada día surgen nuevos Parques de Atracciones y Temáticos (como el que se está realizando en San Martín de la Vega). Imnumerables son también los de las viviendas privadas o los parques públicos, que aumentan progresivamente. Sin embargo, si han desaparecido o han cambiado de función casi todos los Reales Sitios, que surgieron, entre los siglos XVI $y$ $X I X$, como consecuencia de que Madrid fuera elegida, en 1561, capital del reino por Felipe II. Ésta es la causa de que nuestra Comunidad, siendo la cuarta más pequeña de España, cuente con casi la mitad de los jardines declarados históricoartísticos en nuestro país.

\footnotetext{
* Profesora Titular de la Escula Técnica Superior de Arquitectura. Universidad Politécnica de Madrid.
} 
No quisiera que el título de este artículo diera la impresión de que la Comunidad de Madrid cuenta en nuestros días con menos jardines de los que ha tenido a lo largo de su historia, puesto que la evolución sufrida es todo lo contrario. Afortunadamente, las zonas verdes, tanto públicas como privadas, que pueblan nuestro territorio han ido aumentando progresivamente, salvo en el apartado de los Reales Sitios, ya que o se han perdido o se han convertido en parques abiertos al público.

La finalidad de este trabajo es dedicar un recuerdo a algunos de estos importantes jardines que se hicieron en el ámbito de la Comunidad, tanto en el plano privado (viviendas, Reales Sitios) o en el público (Jardines Botánicos, de Recreo, plazas ajardinadas $u$ otras zonas verdes públicas). El criterio elegido para su exposición es el cronológico, ordenándolos así en cada apartado.

No serán objeto de nuestro interés los jardines que han visto cambiado su trazado original, de los que, a modo de ejemplos, damos los siguientes casos: Jardín que estaba delante de la fachada principal del palacio de EI Pardo, existente ya en el siglo XVII, en el que se daba el estilo renacentista, regular y flanqueado por túneles de verdura. En el siglo XIX fue transformado en un jardín de frutales y el la actualidad presenta un eje principal, flanqueado por un variado arbolado y que lleva a dicho edificio. También ha sucedido en el Jardín del palacio de La Zar-

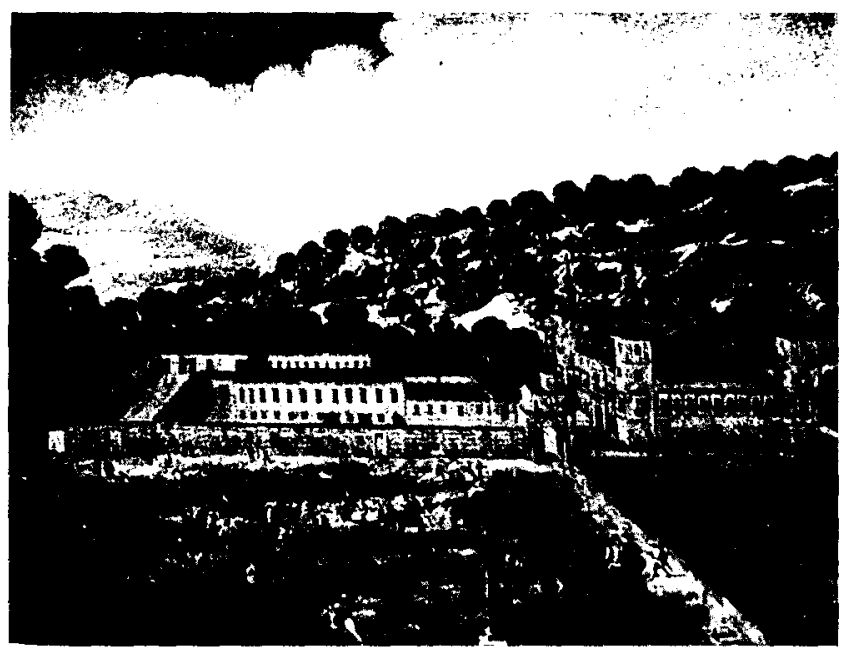

Fig. 1. Primitivo Palacio del El Pardo, cuyo jardín principal está hoy modificado. (Pintura anónima del siglo XVII. Instituto Valencia de Don Juan). 
zuela, que se componia de dos terrazas, unidas por un eje principal, que partía del sencillo palacio, obra de Juan Gómez de Mora. Un muro de contención separaba los dos niveles: el superior ocupado por jardín regular, compuesto por diversos cuadros de boj, llenos de flores, mientras que el inferior se dedicó a huerta, en la que eran importantes los frutales. En el siglo $X X$, después de reformarlo para ser la residencia de los entonces Príncipes de España, en lugar de los tradicionales setos, se plantaron en la terraza superior praderas, junto con arizónicas, pinos, acabuches, etc.; la inferior se ve igualmente cubierta de pradera, salpicada con macizos de flores.

También ocurrió en la terraza superior del Jardín Botánico de Madrid, en la que su primitivo y sencillo trazado regular fue cambiado, en la segunda mitad del siglo del siglo XIX, por otro más complicado y sinuoso muy del gusto de la época isabelina, que es el que podemos contemplar en la actualidad. Simultáneamente, en el centro de esta plataforma se ubicaba un estanque en el que se erigía un busto en honor del botánico sueco Linneo.

Igualmente, se modificó el diseño del Campo del Moro de Madrid, situado a los pies de la fachada occidental del Palacio Real y mandado ajardinar, en 1844, por Isabel II a Narciso Pascual y Colomer ${ }^{1}$, que le dió un trazado hipodámico, a base de paseos paralelos y perpendiculares, en cuyos cruces se formaban pequeñas plazoletas semicirculares y circulares. Entre ellos quedaba un frondoso arbolado, entre el que se colocaron dos bellas fuentes, traídas de otros Reales Sitios: la de las Conchas y la de los Tritones.

Sería Mª Cristina de Habsburgo-Lorena la que decidia reformarlo hacia 1890, encargando la obra al jardinero catalán Ramón Oliva, quien haría una profunda transformación, cambiando el anterior diseño por uno de estilo paisajista, aunque marcando levemente un eje $\mathrm{N}-\mathrm{S}$, a la vez que dejaba las dos fuentes mencionada en el eje E-W, en el que a mediados de nuestro siglo se trazaría el amplio paseo que hoy vemos, también la adornaría con la Casa de Corcho y el Chalet del Rey

Pero entremos en los jardines que nos interesan para este artículo: son los que han desaparecido. De éstos distinguiremos los que aún conservan algún elemento o queda el lugar donde estuvieron , para terminar con los que se han perdido en su totalidad.

1 ARIZA MUÑOZ, CARMEN: Los jardines de Madrid en el siglo XIX, Madrid, Avapiés, 1988, p.103 
A) En este primer apartado vamos a tratar sobre algunos importantes jardines que han desaparecido, pero de los que se conserva el recinto donde estuvieron, por lo que podemos hacernos una mejor idea de su tamaño y otras características.

Uno de ellos es EL JARDÍN DEL FOSO, que se hallaba a los pies del PALACIO DE EL PARDO, mandado hacer por Felipe II, rodeando la construcción ya existente. Su autoría se atribuye a Juan Bautista de Toledo, seguramente siguiendo los consejos del propio monarca, quien lo disfrutaba desde las ventanas del palacio, ya que desempeñaba el papel de una alfombra tendida a sus pies. Como era habitual, presentaba un trazado regular "muy agradable, de muchas verduras, arrayhanes, murtas, gran diferencia de yerbas y flores raras y exquisitas de gran olor y fragancia" según lo describe López de Hoyos en 1572. Su aspecto se puede completar con las palabras de Argote de Molina: "Éntrase en la Casa por dos Puentes de piedra... y en torno una ancha cara y en el fondo de ella muchos compartimentos, vasos y macetas de hierbas medicinales y flores extrañas traidas de diversas regiones, adornadas las paredes de la

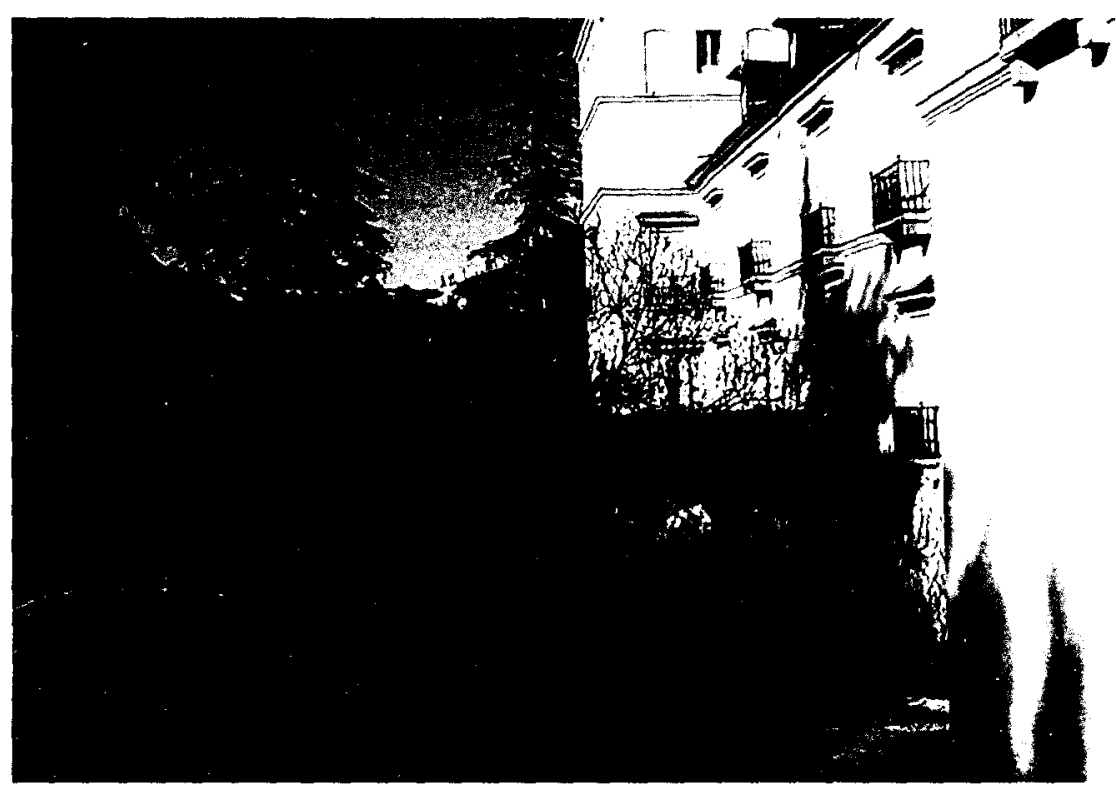

Fig. 2. - Estado actual del foso, que rodea el Palacio de El Pardo. En este lugar mandó hacer Felipe Il el llamado Jardín del Foso. 
cava con jazmines, yedra y rosas y en cada esquina una fuente de agua, que por mascarones de piedra sale»2.

A pesar del incendio del palacio, acaecido en 1604, este jardín contiuaría existiendo, no pudiéndose decir lo mismo a comienzos del siglo $\mathrm{XIX}$, en que estaba prácticamente arruinado, aunque hubiera habido diversos intentos para ajardinarlo. Hubo que esperar a 1814 para que se hiciese realidad, si bien ya presentaría un distinto carácter, puesto que fue convertido en un huerto de frutales, entre los que abundaban los avellanos, granados y perales, no faltando tampoco las hortalizas y flores. Actualmente se han plantado algunos frutales, viéndose también hiedra sobre sus muros en talud.

Igualmente fue Felipe II el impulsor del nacimiento del primer Real Sitio de la capital del reino: la CASA DE CAMPO, en la que se conserva muy poco de lo que ha habido a lo largo de su historia, que como posesión de la Corona se remonta al año 1556, año en el que el aún príncipe, ordenaba comenzar la compra de las tierras situadas al otro lado del río Manzanares, con el fin de formar un gran "parque» que prolongara los terrenos que se hallaban a los pies de la fachada occidental del antiguo Alcázar.

El jardín más importante del lugar estaba en el llamado RESERVADO, hecho sobre una de las propiedades adquiridas: la finca de los Vargas, que tenía una modesta casa-palacio, aprovechada por el monarca. Sus muros eran de ladrillo y cubierta de teja, mientras que sus fachadas aparecian abiertas por galerías de columnas graníticas toscanas en las que se apoyaban arcos de medio punto. Desgraciadamente, en su lugar hoy vemos otra construcción, que aún contiene algunos de los mencionados soportes.

A la vez que se llevaban a cabo importantes labores de infraestructura (canalizaciones de aguas, construcción de presas, nuevas plantaciones, etc.), se trabajaba activamente en el mencionado Reservado ${ }^{3}$ o zona más intima, habitual en este tipo de posesiones, separada por sus propias tapias del resto del recinto y en la que se encontraban, junto a la residencia, los jardines más refinados. En este caso, se plantaron a los pies de su fachada septentrional los tipicos parterres bajos y cuadrangulares, que contenían una rica variedad de flores. Al igual que en los jardines de Aranjuez, en ellos confluian las influencias venidas de Italia y Flandes, sin

2 TOVAR MARTIN, VIRGINIA: EI Pardo, Madrid, Avapiés, 1994, pp.36-37

3 TEJERO VILLARREAL, BEATRIZ: Casa de Campo, Madrid, Avapiés, 1994, pp. 20-23 


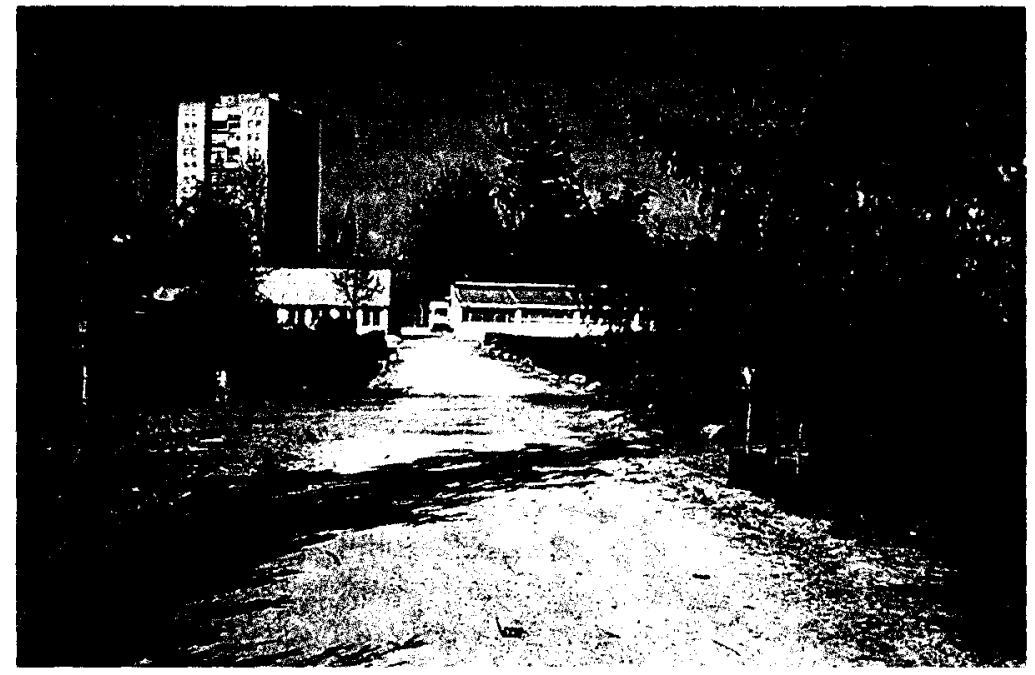

Fig. 3.- Estado actual del que fuera Reservado de la Real Casa de Campo.

que faltaran los consabidos toques islámicos, puesto que volvian a verse los bancos y fuentes de cerámica.

Aunque en un terreno llano, también aquí se producia, como en las villas italianas, la transición entre arquitectura, jardín, bosquete, parque y paisaje natural. Ya que, inmediato a los jardines, aparecía un frondoso bosquete. Igualmente habia fuentes, de las que destacaba la llamada del Águila, situada en el eje principal y algo metida entre los árboles; se hallaba rematada por el águila bicéfala del escudo de Carlos I, que ya no se conserva, aunque el resto lo podemos admirar en el centro del patio de la Universidad $\mathrm{M}^{\mathrm{a}}$ Cristina del El Escorial. En el mismo eje del Reservado y cerca de ella, se ubicaría posteriormente la estatua ecuestre en bronce de Felipe III, que hiciera Juan de Bologna y que en el siglo XIX fue trasladada a la Plaza Mayor.

Tampoco faltaron en este recinto contrucciones típicas de los jardines italianos, como eran las grutas, como la llamada de Neptuno, en cuyo interior, decorado con bajorrelieves y conchas se encontraban las estatuas del dios del mar, Venus y Diana. Hubo incluso una Sala de Burlas.

Afortunadamente todavía podemos ver la ubicación del que fue el primer JARDIN BOTÁNICO de Madrid, el llamado de MIGAS CALIENTES, mandado hacer en 1755 por Fernando VI, a orillas rio Manzanares, en el 


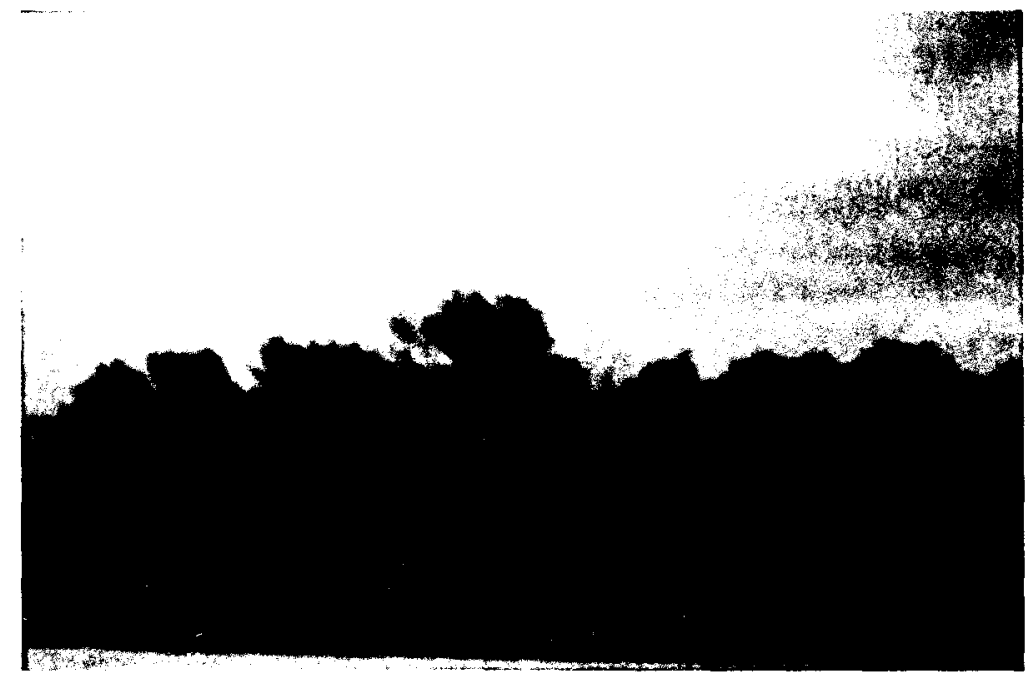

Fig. 4.- Viveros municipales de Migas Calientes, sobre cuya superficie se hizo, en 1755, el primer Jardín Botánico de Madrid.

soto del mismo nombre y hoy ocupado por un vivero municipal. Para su creación se aprovecharía otra huerta de plantas medicinales propiedad de Luis Riquieur4, botánico francés de Felipe $\mathrm{V}$, adquiriendo pronto una destacable frondosidad, a base de los árboles y otras plantas que estaban dispuestos en cuadros. Sin embargo, debido a su lejanía de la capital, empezaría a decaer, pasándose sus plantas al nuevo que se hizo, pocos años después, en el paseo del Prado.

También han desaparecido los jardines que cubrian las terrazas del PALACIO DE BOADILLA DEL MONTE, que perteneciera al Infante Luis de Borbón, hermano de Carlos III, y que realizara Ventura Rodríguez ${ }^{5}$.

Aunque en espera de ser restaurado, el edificio, de ladrillo y piedra, preside el espacio en el que se extendia el jardín, formado por tres niveles. Los dos superiores aparecían ajardinados geométricamente con altos setos de boj. En ella se veían dos amplios espacios cuadrados, en los que se inscribian sendos círculos, completándose el trazado con líneas diagonales y otras curvas. Entre ellos se encontraba la bella fuente de las

4 AÑÓN FELIU். CARMEN: “Noticias sobre los reales Jardines Botánicos de Migas Calientes y de El Prado" Anales del Instituto de Estudios Madrileños T. XXI, 1984, p. 94

5 PONZ., ANTONIO: Viage de España, Madrid, Facsimil De. Atlas, 1972, T.VI, p. 145 
Conchas, también diseñada por dicho arquitecto en 1765 y que hoy se halla en el Campo del Moro, después que fuera regalada a Fernando VII, cediéndola a su vez a su esposa, $M^{a}$ Cristina, quien la instaló en su finca de Vista-Alegre, a la que nos referiremos más adelante. La terraza inferior, más grande, se dedicaría a un importante cultivo de vid y frutales.

Con el paso del tiempo y al ir pasando por diversos propietarios, el lugar iría perdiendo su esplendor y también sus jardines, bien hoy vemos las terrazas y la tapia, junto con el palacio.

En el caso de la CASITA DEL PRÍNCIPE que, en 1784, el futuro Carlos IV mandara hacer a Juan de Villanueva en EI Pardo, hemos perdido la terraza superior de las dos que componian su jardín, presidido por el gracioso Casino, en el que su autor ensayaría la estructura que más tarde dió al Museo de Ciencias (hoy, del Prado).

El diminuto edificio, de ladrillo, piedra y cubierta de plomo, presenta en cada una de sus dos fachadas una entrada diferente: en la oriental o principal se ve el escudo, obra de Pedro Michel, rematando el pórtico jónico «in antis», solución muy característica de Villanueva; de ella partía un paseo de tilos que conducía al Palacio. La fachada occidental es más sencilla, ya que el acceso es un simple vano rematado en medio punto, que da a una pequeña pieza cubierta por una graciosa cúpula de media naranja.

De ella se pasaba al jardín posterior que era el más importante. Así, aprovechando el desnivel del terreno existente desde este punto hacia el cercano río Manzanares, se trazaron dos terrazas enlazadas por una tosca fuente-escalera de granito y por un eje estando ambas totalmente ocupadas por parterres, hechos a base de setos de boj. Sólo se conserva la terraza inferior, cercada por una graciosa tapia, que se encuentra cubierta por complicados y bellos setos de boj.

Durante el reinado de Fernando VII, la capital vería nacer dos nuevos Reales Sitios que, desgraciadamente, han desaparecido, aunque se conserva su perímetro y algunos de los elementos que los componían.

Uno de ellos fue el llamado CASINO DE LA REINA, que se haría sobre la llamada huerta de Romero, de trazado ortogonal y situada junto a la actual glorieta de Embajadores. Fue propiedad de un afrancesado, que llegaría a ser ministro de José I, pasando pocos años después de la marcha de las tropas napoleónicas al Ayuntamiento, que, en 1817, la regalaba a la reina Isabel de Braganza. 


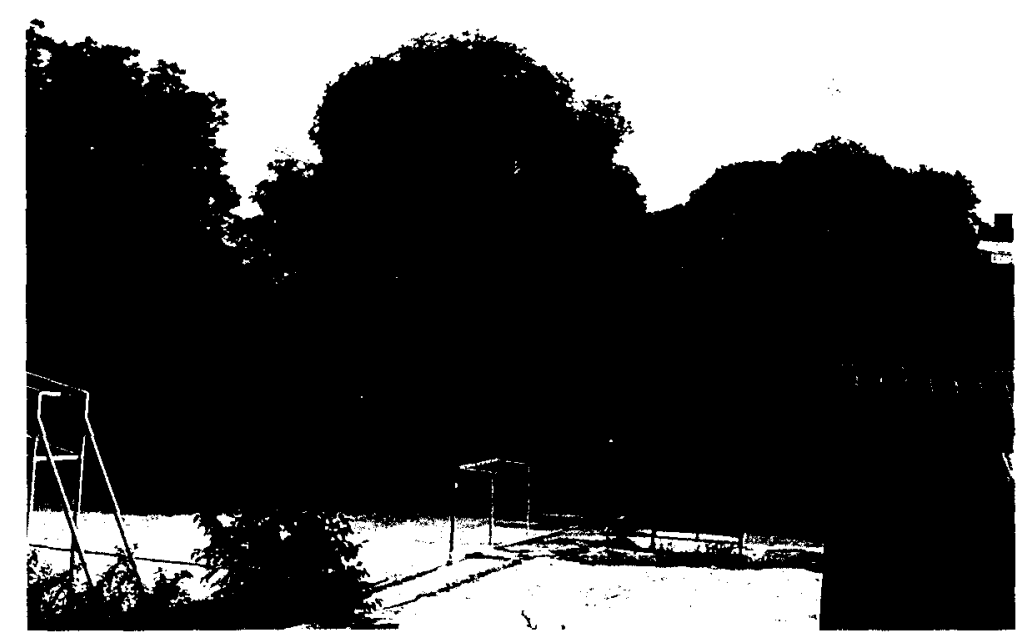

Fig. 5.- Estado actual del que fuera Real Casino de la Reina de Madrid.

Entre los elementos que se aprovecharon se encontaba un sencillo palacete, que aún podemos contemplar y que consiste en un pequeño edificio, compuesto por dos alturas y rematado por un desaparecido chapitel. En esta terraza superior también se hallaban pequeños jardines geométricos y diversas construcciones, de las que destacaba un invernadero de fábrica, recogido en un grabado por Fernández de los Ríos6.

En el resto de la superficie se realizaría un pequeño parque romántico de caminos curvos, aunque mezclados con algunos rectos, en ocasiones cubiertos con pérgolas. Tampoco faltaba la ría navegable de irregulares perfiles y surcada por rústicos puentes, que dejaba en su interior una isleta, además de un gracioso embarcadero en uno de sus extremos, que constaba, según Madoz, de: «... tres cuerpos concéntricos circulares, siendo el más de arriba un templete de doce columnas dóricas de madera y cuatro arcos de medio punto, rematado por un cascarón»?.

También había cenadores, una gruta artificial junto a la ría y una casa rústica. Además de estos, existieron un destartalado invernadero y diver-

6 FERNÁNDEZ DE LOS RÍOS, ANGEL: Guia de Madrid, Madrid, Facsimil Ediciones Ábaco, 1876, p. 454

7 MADOZ, PASCUAL: Diccionario geográfico-histórico y estadístico de España y sus posesiones de Ultramar, Madrid, Imprenta del Diccionario Geográfico, 1847, T.X. p. 909 
sas construcciones de servicio, así como esculturas adornando los pequeños jardines regulares inmediatos al palacete, sin olvidar la elegante entrada, obra de López Aguado8, compuesta por dos grandes pilares con columnas dóricas adosadas y rematados por graciosos grupos escultóricos, con gran semejanza a la que Juan de Villanueva hiciera en el Jardín del Príncipe de Aranjuez.

El Casino tuvo un importante papel recreativo, hasta que Isabel II lo cediera al Estado, para instalar, desde en 18719 y junto a la glorieta de Embajadores, el Museo Arqueológico Nacional, trasladado en 1895 al actual, mientras que Francisco Jareño, en estilo neomudéjar, realizaba la Escuela de Veterinaria, hoy ocupada por el Instituto Cervantes 10. Actualmente, ha terminado su restauración, habiéndose abierto al púbico, con lo que Madrid ha ganado un nuevo espacio verde, que conserva algún elemento de su interesante pasado, como algunos árboles y el mencionado palacete.

El otro Real Sitio fue el de VISTA-ALEGRE en Carabanchel, cuya creación sería impulsada por $\mathrm{M}^{\mathrm{a}}$ Cristina de Borbón a lo largo de la década de 1830, durante la que se llevaron a cabo las obras, convirtiéndose en el sustituto de la Malmaison de Josefina, de la que habían sido propietarios tras la muerte de la que fuera esposa de Napoleón.

Un equipo, formado por el aparejador José Petorelli y más tarde por el arquitecto Martin López Aguado, asi como por el jardinero Santos Antolín, entre otros, llevaría a cabo un amplio jardín paisajista, survado por dirversos arroyos y una ría artificial navegable, que partía de una isleta circular "... en forma de pequeña fortaleza rotonda con sus baluartes"11. Junto al extremo opuesto de esta vía de agua se levantaba la Montaña Artificial de rocalla, que estaba rematada por un templete clásico de madera y de la que salía una cascada.

Como era habitual en este tipo de jardines románticos, además de la mencionada Montaña, había otros "caprichos" arquitectónicos, como una Faisanera, una Pajarera, Naranjera, el llamado Castillo Viejo, una

8 NAVASCUÉS PALACIO, PEDRO: Arquitectura Española (1808-1914), Madrid, Espasa-Calpe,1993, T. XXXV Summa Artis, p. 131.

9 ARIZA MUÑOZ, CARMEN: “Un Real Sitio madrileño desaparecido" Zona Verde otoño 1992, p. 18

10 ARIZA MUÑ̃Z, CARMEN: Los jardines de Madrid en el siglo XIX, Madrid, Avapiés, 1988, pp. 86-96

11 RODRIGUEZ ROMERO. EVA: El jardin paisajista y las quintas de recreo de los Carabancheles: la posesión de Vista-Alegre, p. 790 de la Tesis Doctoral, leida en julio de 1999 en la E.T.S. de Arquitectua de Madrid. 


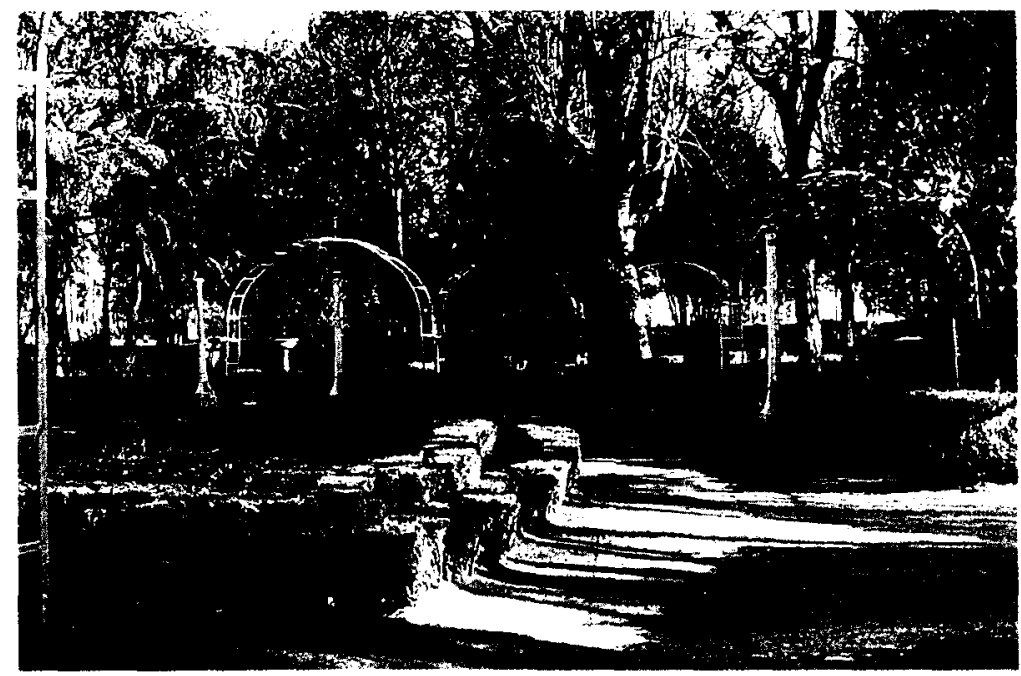

Fig. 6. - Jardín restaurado en el que fuera Real Sitio de Vista Alegre, situado en Carabanchel.

Casa de Vacas, etc. ${ }^{12}$. Metidas entre un variado arbolado (de los que aún quedan cedros, eucaliptos, etc.), se veían diversas fuentes, siendo la más destacable la ya mencionada de las Conchas, que fue traída del palacio de Boadilla del Monte y luego trasladada al Campo del Moro.En cambio, si podemos ver actualmente los dos palacios. El primero de ellos, llamado Viejo, hecho tras aprovechar uno ya existente, se halla situado a lo largo del paseo principal de la posesión, ya que es un extraño edificio-pantalla, de dos alturas formadas por amplios vanos adintelados entre las columnas toscanas del piso bajo y las jónicas del superior. Junto a él habia un parterre regular, con paseos radiales, que ha vuelto a reconstruirse.

A su lado, afortunadamente, encontramos la Estufa u "orangerie", compuesta de una rotonda central, apoyada sobre columnas pareadas toscanas, que sostienen un sobrio entablamento y una pequeña cúpula. Está flanqueada por dos piezas de planta rectangular, formadas por el mismo tipo de soportes y vanos adintelados ${ }^{13}$. El otro palacio que, aunque muy deteriorado, se sigue conservando, era el Nuevo, quizás obra de Martín López Aguado. 
En 1846 Vista-Alegre era de Isabel II y de su hermana Luisa Fernanda, quien la compraba en 1858. Al año siguiente, pasaba al marqués de Salmanca, que mandaría a Narciso Pascual y Colomer remodelar el palacio Nuevo, delante del que se haría el pequeño jardín, con la llamada fuente de los Caballos, rodeada de parterres de tipo isabelino y que hoy podemos ver en un lamentable estado. Igualmente se llevaría a cabo una importante estancia: la denominada, y lamentablemente desaparecida, Sala de Fumar, realizada por el restaurador-adornista de la Alhambra de Granada, Rafael Contreras, que le daria el consabido estilo neonazarita, como era habitual en otros palacios regios, como es el caso del de Aranjuez ${ }^{14}$.

Los herederos del marqués venderían la posesión al Estado, que instalaría en ella una serie de establecimientos de carácter benefico, algunos de los cuales se mantienen, juntos con otros organismos, concretamente de la Comunidad de Madrid.

Aunque en el capítulo de la jardinería urbana es en el que menos jardines se han perdido, sí ha desaparecido la primera zona verde pública que tuvo la capital, la conocida por LA TELA, situada junto al límite meridional del Campo del Moro. A mediados del siglo XIX, cuando se ajardinó el parque del Palacio Real, el Ayuntamiento decidía embellecer su propiedad, conviertiendo el lugar en un bosquete de árboles y arbustos de hoja perenne, dispuestos según una simple diseño, ya que la superficie aparecia atravesada por dos paseos rectos orientados de N-S y E-W, que se cruzaban en el centro formando una plazoleta circular, según puede verse en el plano de F. de Coello de 184815. Con los años este espacio se deterioraría, encontrándose hoy sobre su superficie el actual parque de Atenas.

Tampoco conservamos algunas pequeñas PLAZAS AJARDINADAS, tipo square de gusto isabelino, realizadas en Madrid desde 1860. Entre ellas se encuentra la de SANTO DOMINGO, hecha según el modelo inglés con césped, numerosos cedros, magnolios, rododendros, asi como arbustos y flores. Todo ello rodeado por una sencilla verja de hierro, diseñada por el entonces arquitecto municipal Félix $\mathrm{M}^{\mathrm{a}}$ Gómez. La superficie verde desaparecería en 1959 para construir un aparcamiento subterráneo.

En el centro la PLAZA MAYOR de Madrid, que concibiera en el siglo XVII Juan Gómez de Mora y que restaurara más tarde Juan de Villanueva, se plantaba un jardín, en la segunda mitad del siglo XIX, siguiendo el

14 NAVASCUÉS PALACIO, PEDRO: Un palacio romántico. Madrid (1846-1858), Madrid, Ediciones EI Viso, 1983, pp. 86-88

15 ARIZA MUÑOZ, CARMEN: Op. cit, p. 227. 


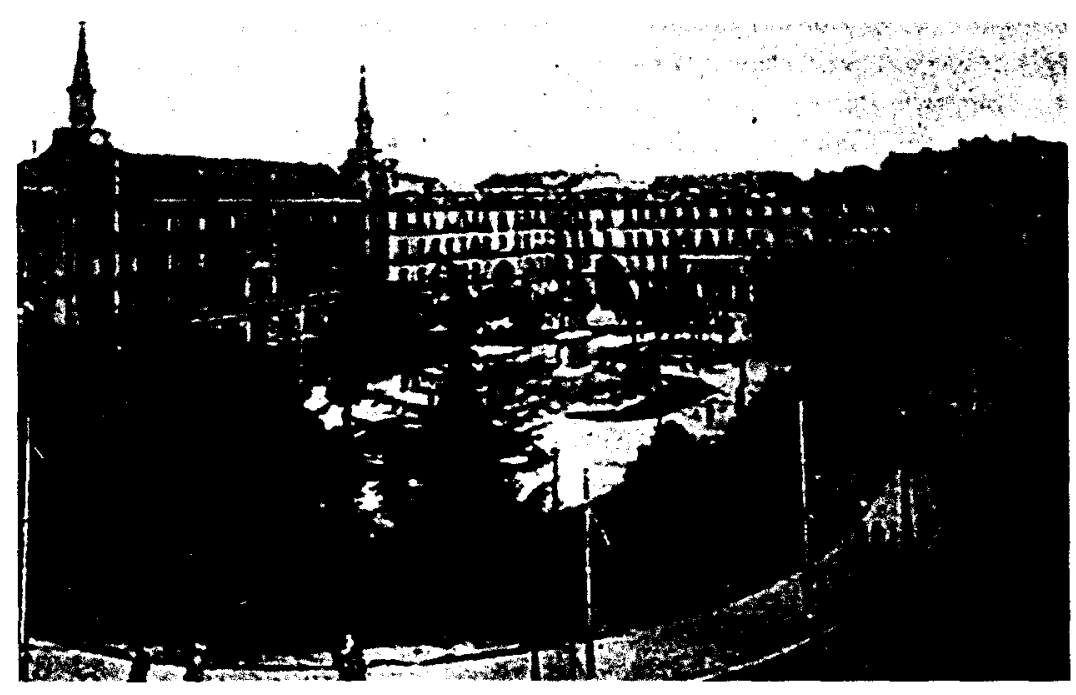

Fig. 7.- Desaparecido jardín de la Plaza Mayor de Madrid.

ejemplo de lo que se hacía en otras capitales del mundo occidental. Sólo queda en la madrileña la mencionada estatua ecuestre de Felipe III, que se habia trasladado a este punto procedente de la Real Casa de Campo. El jardín desapareció a mediados del siglo XX.

Como veremos más adelante, la capital ha perdido todos lo JARDINES DE RECREO que tuvo durante los siglo XIX y XX. Sin embargo, en el actual parque de la Fuente del Berro estuvo instalado el llamado NUEVOS CAMPOS ELISEOS, que aprovechó, durante pocos años el actual palacete para ubicar el restaurante. Sin embargo, no se conservan otros elementos, como invernaderos, una torre-mirador, un invernadero, un velódromo, una montaña rusa, etc. ${ }^{16}$. así como algunas zonas ajardinadas.

B) Este segundo apartado lo dedicamos a algunos jardines que se han perdido en su totalidad, hasta tal punto que no se conserva ni su recinto o marco en los que estuvieron, al estar en su mayor parte edificados.

Entre ellos cabe mencionar dos antiguos y modestos Reales Sitios, realizados en el siglo XVI en los alrededores de la villa de Madrid. Uno es el de $A C E C A$, hecho sobre unos terrenos que Carlos I, en 1534, incorporara al Real Bosque de Aranjuez procedente de una encomienda de los ma- 


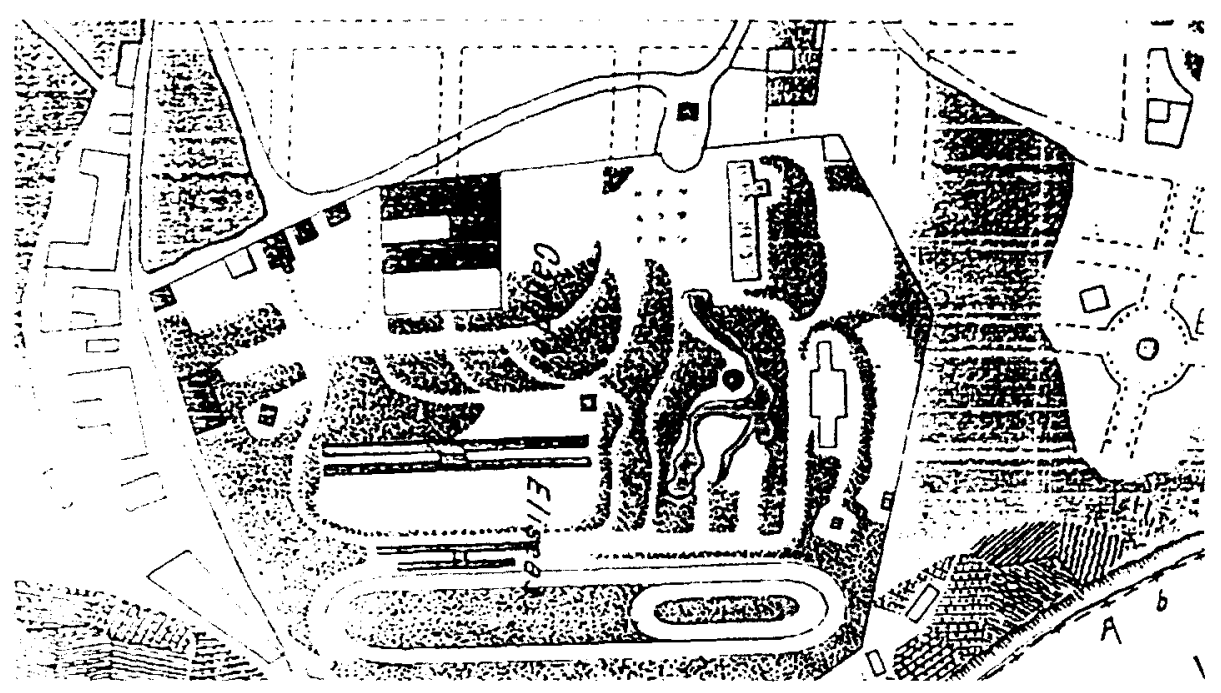

Fig. 8. - Plano del Jardín de Recreo denominado Nuevos Campos Elíseos, sobre cuya superficie se encuentra hoy el parque de la Fuente del Berro de Madrid.

estres de la Orden de Calatrava y que estaba situada en el camino de esta población ribereña a Toledo.

Alli habia existido un castillo, remodelado en tiempos de Felipe II, resultando unas construcciones hechas con muros mixtos, esto es, a base de ladrillos y una irregular mampostería, siendo las cubiertas de teja. Si bien no conocemos la existencia de algún jardín, en un óleo del siglo XVII aprarecen unas sencillas tapias, que pudieran haberlo cobijado, como era habitual en esta época.

El mismo monarca también mandaba comprar unos terrenos, situados en la confluencia de los ríos Manzanares-Jarama y frente a las peñas llamadas de Cuelgamujeres 17. En ellos haría otro modesto Real Sitio, denominado VACIAMADRID. En otro lienzo del Siglo de Oro puede verse una pobre construcción del mismo estilo que la anterior. Junto a ella e igualmente limitado por semejantes tapias, aparece claramente un pequeño jardín plano, compuesto por una fuente rodeada de veinte parterres cuadrados de boj con abundantes flores, dispuestos ortogonalmente, al igual que los que se llevaron a cabo en el Reservado de la Casa de

17 TEJERO VILLARREAL, BEATRIZ: “La Casa Real de Vaciamadrid» en Jardín y Naturaleza en el reinado de Felipe II, Madrid, Ediciones Doce Calles, 1998, p. 513 
Campo.Tras la muerte del rey, el lugar entraria en una profunda decadencia, hasta que dejó de ser de la Corona en el siglo XVIII, no conservándose ningún vestigio de la misma.

Una de las posesiones regias más emblemáticas en la capital fue el ALCÁZAR surgido de aprovechar y ampliar el edificio que levantara Muhamad I a mediados del siglo IX y que los reyes Trastámara seguirían utilizando durante sus frecuentes jornadas cinegéticas por los alrededores de Madrid. En 1734, era totalmente destruído por un incendio, sirviendo su solar para que el primer monarca Borbón, Felipe V, mandara levantar el actual Palacio Real.

Tampoco se conservan los diversos jardines que adornaban este sobrio castillo-palacio. Uno de ellos era el Jardín del Rey, auténtico "giardino segreto", consistente en un pequeño recinto cerrado por sus propias tapias y tendido al pie de la llamada Torre Dorada, que de levantaba en el ángulo NW del edificio. Su trazado obedecía al consabido módulo cruciforme, compuesto por cuatro parterres rodeando una fuente central; tras ser reformado en el siglo XVII, pasó a llamarse de los Emperadores, por los veinticuatro bustos de mármol de emperadores romanos, más el de Carlos I, que lo decoraban.

A los pies de la fachada oriental se haría el Jardín de las Infantas, más tarde llamado de la Reina, de mayor extensión que el anterior pero con el mismo esquema cruciforme. Junto a la parte norte del palacio se encontraba el denominado Jardín del Juego de Pelota, «... que durante el reinado de Felipe IV adquirió su forma y nombre definitivos: el Jardín de las Bóvedas:18, y que estaba a las misma altura que el de la Reina.

En un nivel más bajo que estos se hallaba el llamado Jardín de la Priora o Nuevo, que era el más extenso e importante, ya que ocupaba una amplia zona situada el NE del edificio, justo al norte de la Casa del Tesoro. También se organizaba en cuarteles, adornados con diversas fuentes y un estanque. La mayor parte de los elementos verdes que lo componian eran hortalizas y frutales. Por encima de él, y con la misma disposición ortogonal, se veía la Huerta Nueva, en la que, ya en 1598, existía un recinto dedicado a plantas medicinales, puesto que "...se cultivaban yerbas para abastecer las reales boticas, conforme proyecto de Honorato Pomar, médico de Felipell/»19. Completaba este cinturón verde en torno al Alcá-

18 SANCHO, JOSÉ LUIS: Jardines de Palacio, Madrid, Patrimonio Nacional, 1994, p. 13

19 MARQUESA DE CASA.VALDÉS: Jardines de España, Madrid, Aguilar, 1973, p. 170 
zar el llamado Parque, situado al pie de su fachada occidental, donde hoy se halla el Jardín del Campo del Moro.

Lamentable fue también la pérdida del REAL SITIO DE LA FLORIDA, de la que no conservamos prácticamente nada, salvo algún elemento aislado, como es la ermita de San Antonio del mismo nombre, que hiciera Francesco Sabatini a finales del siglo XVIII, y decorara Francisco de Goya con frescos, que aún hoy pueden contemplarse 20.

Su nacimiento se sitúa en 1792, cuando Carlos IV compraba a la marquesa de Castel-Rodrigo la antigua posesión del mismo nombre, que se componia de un palacete y diversas construcciones de servicio. Lógicamente, no faltaban diversos jardines, adornados con estanques, grutas y fuentes. Igualmente, habia amplias huertas y tierras de explotación agrícola21. A este núcleo inicial se irian sumando otras posesiones, entre las que destacaban las llamadas Montaña del Principe Pio y la de La Moncloa (que habia pertenecido a la XIII duquesa de Alba).

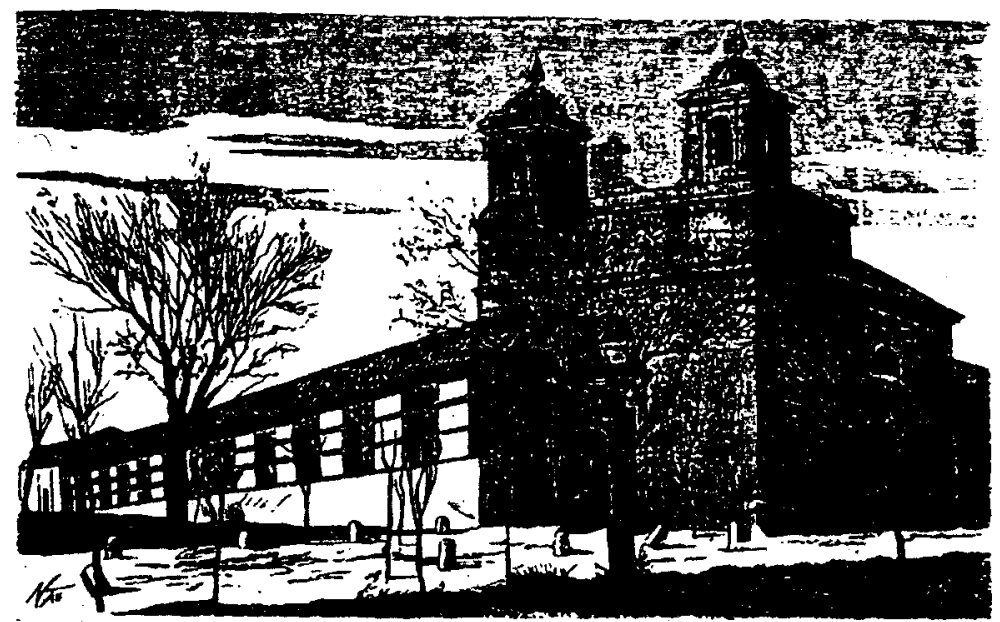

Fig. 9. - Iglesia del desparecido Real Sitio de La Florida.

20 FERNÁNDEZ TALAYA, Ma TERESA: El Real Sitio de la Florida y la Moncloa, evolución históri-co artística de un lugar madrileño, Madrid, Ayuntamiento de Madrid, 1999, p. 232

21 ARIZA MUÑNZ, CARMEN: Los jardines de Madrid en el siglo XIX, Madrid, Avapiés, 1988, pp. 3536 
Tras la invasión de las tropas napoleónicas, Fernando VII hubo de reconstruir esta posesión, de la que se restauraron diversos edificios y "caprichos", entre los que se encontraban la llamadas Casas del Duende o la del Choricero.

Una importante obra de Fernando VII fue el intento, a partir de 1817, de volver a reconstruir en este lugar la recien desaparecida Fábrica de Porcelana, que se hallaba en el Buen Retiro. Para ello trasladaría los pocos restos que quedaban, así como su personal. El establecimiento funcionaría a lo largo del siglo, haciéndose importantes obras cerámicas, que decoraron muchos edificios madrileños. Actualmente, pueden verse en el parque del Oeste algunos restos, un horno de ladrillo en forma de tinaja y una chimenea. Su sucesora, Isabel II, continuaría mejorando La Florida, mandando realizar diversos jardines, e incrementando el arbolado, tanto de sombra como frutales.

Desgraciadamente, tras la Revolución de 1868, la Corona perdería esta propiedad, sobre la cual se levantaron diversos barrios (como el de Argüelles), importantes edificios (el Asilo $M^{a}$ Cristina, el Hospital de la Princesa o la Cárcel Modelo) y, a comienzos del siglo XX, el mismo parque del Oeste.

Muchos son los jardines de las VIVIENDAS PRIVADAS de la Comunidad que podríamos enumerar; sin embargo, a modo de ejemplo, sólo nos referiremos a unos pocos.

Lamentablemente, no conservamos nada de las numerosas viviendas de la capital, que tenían sus zonas de jardines o huertas regulares, encerrados dentro de terrosas tapias, como eran los de las residencias del marqués de Alcañices, del de Medinaceli, del conde de Maceda, etc, extendidas a lo largo del paseo del Prado22.

Otro ejemplo fue la llamada casa-palacio que los duques de Osuna habian adquirido en la zona de Leganitos, en la que, en el siglo XVII, ya existía un jardín de tipo clasicista, en el que se veían balaustradas; fuentes; esculturas de mármol en hornacinas. Tampoco nos ha llegado otra modesta residencia que tenian en las Vistillas y que, en 1830, presentaba un jardín, en desnivel con paseos curvos.

22 SOUTO ALCARAZ, ANGELA: Paisaje urbano del Paseo del Prado: desde la reforma hasta la desaparición del Retiro (1767-1865), pp. 467-479 de la Tesis Doctoral, leida, en 1995, en la E.T.S. de Arquitectura de Madrid. 


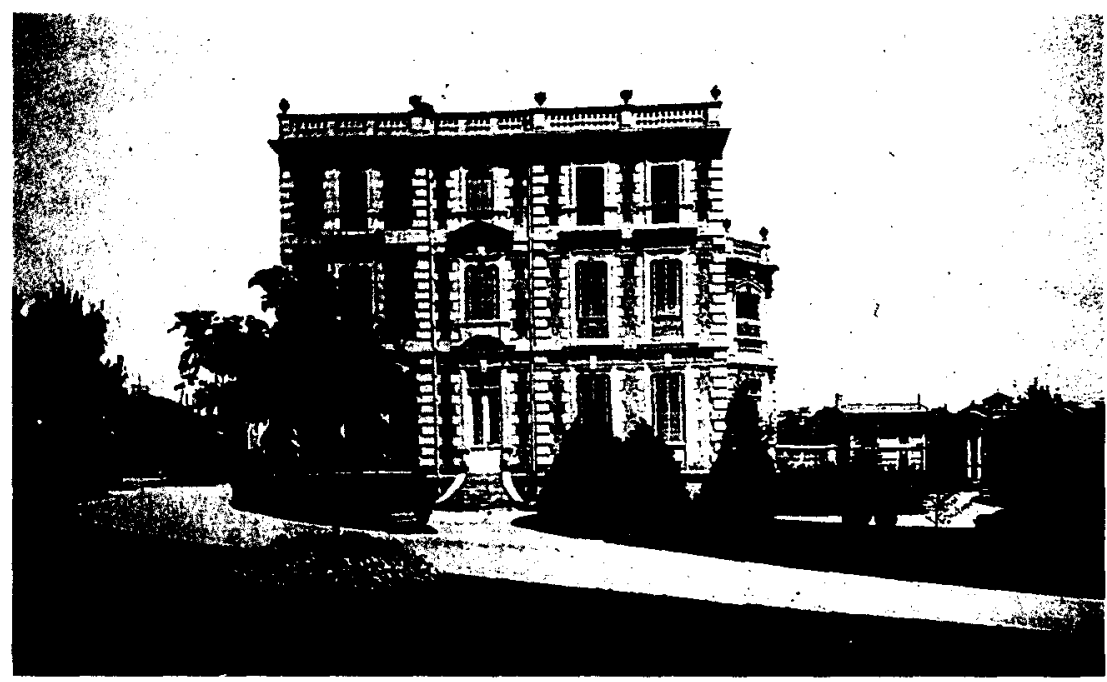

Fig. 10. - Palacio de Indo, uno de los muchos realizados, en el siglo XIX, a lo largo del madrileño paseo de La Castellana.

De los elegantes palacios realizados en el siglo XVIII, Liria, Buenavista o de Villahermosa, no conservamos el jardín de este último, que debía de tener un trazado a la inglesa, con un estanque, el consabido puente rústico, el cenador chinesco, etc., todos ellos en medio de un frondoso arbolado23.

Más modestos fueron los jardines que la burguesía madrileña decimonónica realizaría rodeando sus palacetes y hoteles urbanos, construídos con los más diversos estilos arquitectónicos, de los que la mayor parte han desaparecido, como el neonazarita palacio Xifré, la quinta de los Pajaritos, la posesión conocida por La Huerta (propiedad de Cánovas del Castillo, ubicada donde hoy se halla la Embajada de los Estados Unidos de América), el palacio de Indo, entre los muchos que flanqueaban el gran eje verde de la capital, formado por los paseos del Prado, Recoletos y la Castellana. Aunque no se conserven los jardínes y nos han quedado algunos de los edificios, como el de Linares; el que fuera hecho por Narciso Pascual y Colomer para el marqués de Salamanca y hoy es sede bancaria; etc. Igualmente, los palacetes y hoteles abundaban por otras zonas de la capital, como en

23 NAVASCUÉS PALACIO, PEDRO: “Casas y jardines nobles de Madrid” en Jardines Clásicos Madrileños. Catálogo de la exposición celebrada en el Museo Municipal de Madrid en 1981, Madrid, Ayuntamiento de Madrid, 1981, pp. 121-122 
el recientemente construído barrio de Argüelles, o en la nueva y verde Ciudad Lineal.

Simultáneamente, en algunos pueblos de las inmediaciones de la capital se fueron levantando un buen número de quintas o villas suburbanas, que especialmente eran abundantes en los Carabancheles. Vecinas al Real Sitio de Vista-Alegre, la nobleza y la burguesía madrileña irian poblando estos de propiedades, a las que iban en determinadas épocas del año. Entre ellas podemos mencionar la posesión de José Gargallo (con bellos paseos arbolados y abundantes frutales), o las quintas del marqués de Remisa, de Manuel Mateu (cuyo jardín tenía una casa rústica de claro sabor romántico), la llamada Delicias Cubanas (con una bella estufa), la de Miranda o de casa de Montijo (con extensas superficies de viñas, olivares y también algunas zonas ajardinadas y de bosquetes) ${ }^{24}$. De algunas de ellas restan edificos, utilizados para otros fines 25 .

De los numerosos jardines que tenía el Palacio de La Moncloa, que fuera de la duquesa de Alba, $\mathrm{M}^{\mathrm{a}}$ Pilar Teresa Cayetana de Silva, se han perdido los llamados de la Princesa, Parterre, Caño Gordo, Paso, Estufa, Laberinto. Sin embargo, sí existe el denominado del Barranco.

El primero era descrito por Winthuysen como: «El jardín de la Princesa es un resto de parterres más extensos, que fueron arrancados, y está formado por bojes que forman una traza sencilla y un tanto monótona, compartidos en cuadros, y en medio una glorieta con una fuente de piedra, en cuyo centro hay un pedestal con su taza: en torno se desarrollan grandes abetos, y hay también en los recuadros secuoias gigantes, especies introducidas,... en la segunda mitad del siglo XIX»?26.

De los otros espacios verdes dice: “El llamado jardín del Parterre es semejante al anterior". "El del Caño Gordo formaba una bella composición: se desciende a él por dos rampas, que se combinan a trozos con escalones, $y$ en sus antepechos, pedestales para vasos. En el espacio en forma de trapecio que queda entre estas rampas, un parterre de plano inclinado, de bonito dibujo, y allí brota la fuente de Caño Gordo. A la bajada hay un estanque rodeado de pedestales y baranda de hierro, que deja,

24 ARIZA MUÑOZ, CARMEN: Op. cit. pp. 130-134

25 RODRIGUEZ ROMERO, EVA: El jardín paisajista y las quintas de recreo de los Carabancheles: la posesión de Vista-Alegre, pp. 522- 555 de la Tesis Doctoral, leída en julio de 1999 en la E.T.S. de Arquitectura de Madrid.

26 WINTHUYSEN, JAVIER DE: Jardines Clásicos de España, Madrid, Facsímil por Ediciones Doce Calles, 1930 , p. 120 
entre ésta y el borde de piedra que encierra el agua, platabanda para flores, y más allá del estanque había otro parterre con un surtidor. Estas plantaciones, que conocimos muy bellas y originales, fueron arrancadas, y en el resto de las otras se hicieron reformas ridiculas, quitando a este gracioso rincón su belleza y marcador carácter".

"En el jardín del Paso quedaba parte de su sencillo parterre, unas grandes figuras de bojes tallados y un estanquillo ovalado de moldura de piedra y surtudor. Otra graciosa fuentecilla, que formaba una cascadita, se arruinó por completo".

"En el jardín de la Estufa quedan unos cuadros de bojes, donde hay grandes coniferas, una fuente central y restos de otra adosada al muro, y la entrada del jardín forma una portada sencilla neo-clásica con escaleras".

"El Laberinto nada consenva de su traza primitiva: sólo existe la fuente, que es de piedra, con una gran taza sobre su columna, y terminada por un ánfora. Forma este jardín ahora un bosque de plátanos".

La mayor parte de los JARDINES DE RECREO que nacieron en Madrid durante el siglo XIX27 han desaparecido. De ellos mencinaremos dos de los más importantes: el llamado Campos Elíseos, situado en la superficie hoy comprendida entre las calles de Alcalá, Velázquez, Hermosilla y Castelló, aproximadamente. Tras pagar dos reales, los madrileños encontraban allí un teatro, sala de conciertos, una pequeña plaza de toros, pistas de patinar, restaurantes, etc, en medio de una zona verde, que no creció lo deseado porque, a los veinte años, fueron destruídos para levantar el barrio de Salamanca.

El otro fue el Jardín de Recreo del Buen Retiro, hecho cuando desapareció el anterior aprovechando una antigua zona verde del desaparecido Real Sitio, que constaría de los mismos servicios, además de un quiosco de música. A pesar de la buena acogida que tuvo también, debiendo pagar sus visitantes una peseta, todo él, junto con su frondoso arbolado, desaparecería a comienzos del siglo XIX, para edificar sobre su solar el actual Palacio de Comunicaciones de la plaza de Cibeles.

Como apuntamos al comienzo de este artículo, aunque se hayan perdido algunos importantes jardines, en la actualidad, la Comunidad de Madrid cuenta con un mayor números de ellos. Sírvannos como ejemplos de 


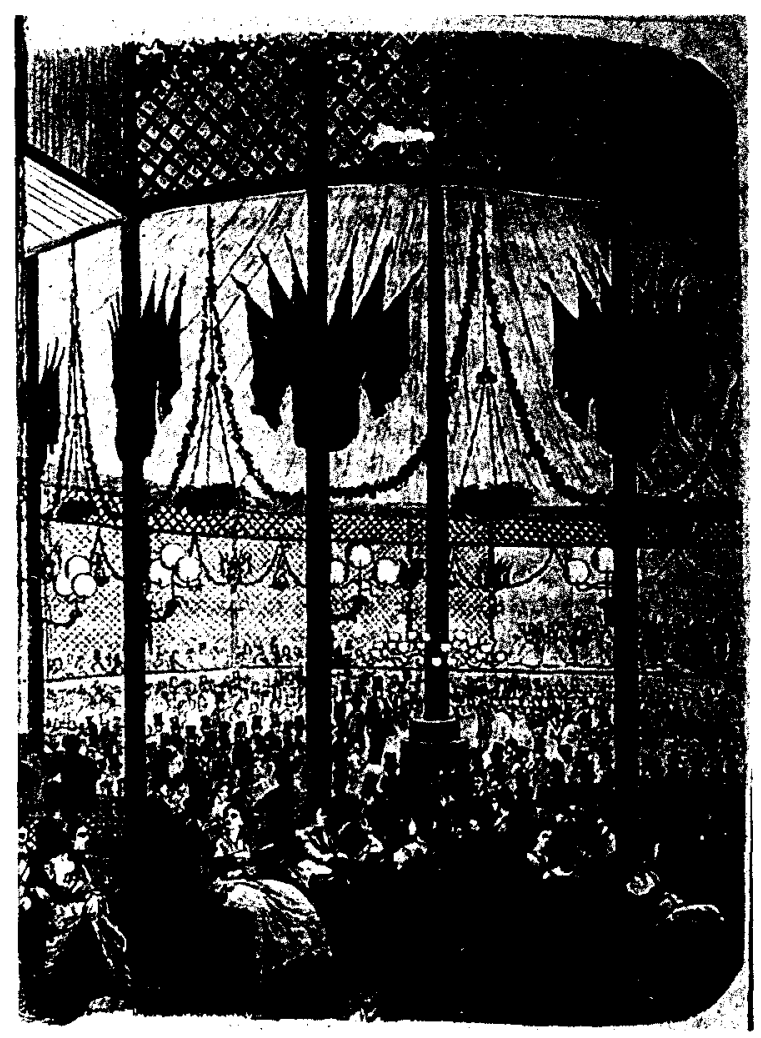

Fig. 11 Salón de Concierto del desaparecido Jardín de Recreo, llamado Campos Elíseos.

los que ya no se conservan el primer jardín Botánico de la capital, pero en cambio existen el del paseo del Prado o los recientes de las Ciudades Universitarias de Madrid y de Alcalá de Henares. Lo mismo podría decir de los Jardines de Recreo, puesto que no nos han llegado los hechos en el siglo XIX, pero cada día surgen nuevos Parques de Atracciones y Temáticos (como el que se está realizando en San Martín de la Vega). Imnumerables son también los de las viviendas y los parques públicos, que aumentan progresivamente. Sin embargo, si han desaparecido o han cambiado de función casi todos los Reales Sitios, que surgieron, entre los siglos XVI y XIX, como consecuencia de que Madrid fuera elegida, en 1561, capital del reino por Felipe II. Ésta es la causa de que nuestra Comunidad, siendo la cuarta más pequeña de España, cuente con casi la mitad de los jardines declarados histórico-artísticos en nuestro país. 


\section{BIBLIOGRAFIA}

AÑon Feliu, CARMEN: «Noticias sobre los reales Jardines Botánicos de Migas Calientes y de El Prado" Anales del Instituto de Estudios Madrileños T. XXI, 1984.

Ariza Muñoz, Carmen: Los jardines de Madrid en el siglo XIX, Madrid, Avapiés, 1988

“Jardines de Recreo en Madrid: Ios llamados Campos Elíseos" Goya ํㅡ 204, 1988.

"Un Real Sitio madrileño desaparecido" Zona Verde otoño 1992.

Fernández de los Rios, Ángel: Guia de Madrid, Madrid, Facsímil Ediciones Ábaco, 1876.

Fernández TALAYA, M.- TeRESA: El Real Sitio de la Florida y la Moncloa, evolución histórico artística de un lugar madrileño, Madrid, Ayuntamiento de Madrid, 1999.

Machin, CaRlos: El Palacio del Infante Don Luis Antonio de Borbón, Madrid, 1999.

MADOZ, PASCUAL: Diccionario geográfico-histórico y estadistico de España y sus posesiones de Ultramar, Madrid, Imprenta del Diccionario Geográfico, 1847, T.X.

MARQUeSA DE CASA-VALDÉS: Jardines de España, Madrid, Aguilar, 1973.

Navascués Palacio, Pedro: Arquitectura Española (1808-1914), Madrid, Espasa- pe,1993, T. XXXV Summa Artis.

Un palacio romántico. Madrid (1846-1858), Madrid, Ediciones El Viso, 1983.

"Casas y jardines nobles de Madrid" en Jardines Clásicos Madrileños. Catálogo de la exposición celebrada en el Museo Municipal de Madrid en 1981, Madrid, Ayuntamiento de Madrid, 1981, pp. 121-122.

Ponz, Antonio: Viage de España, Madrid, Facsímil De. Atlas, 1972, T.VI.

RODRíGUEz, EVA: El jardín paisajista y las quintas de recreo de los Carabancheles: la posesión de Vista-Alegre, p. 790 de la Tesis Doctoral, leída en julio de 1999 en la E.T.S. de Arquitectua de Madrid

SANCHO, José LUIS: Jardines de Palacio, Madrid, Patrimonio Nacional, 1994

Souto Alcaraz, Ángela: Paisaje urbano del Paseo del Prado: desde la reforma hasta la desaparición del Retiro (1767-1865), pp. 467-479 de la Tesis Doctoral, leída, en 1995, en la E.T.S de Arquitectura de Madrid

Tejero Villarreal, Beatriz: Casa de Campo, Madrid, Avapiés, 1994 "La Casa Real de Vaciamadrid" en Jardín y Naturaleza en el reinado de Felipe II, Madrid, Ediciones Doce Calles, 1998

Tovar Martin, Virginia: El Pardo, Madrid, Avapiés, 1994

WinthuYsen, JAVIER DE: Jardines Clásicos de España, Madrid, Facsimil por Ediciones Doce Calles, 1930, p. 120 